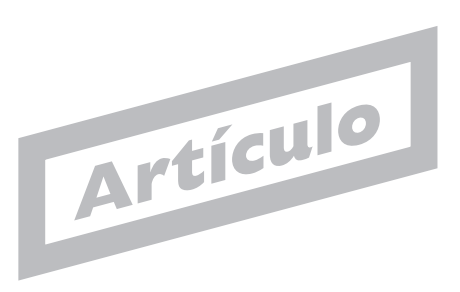

\title{
Del comercio electrónico a la administración electrónica: tecnologías y metodologías para la gestión de información
}

\author{
Por Pablo Lara Navarra y José Ángel Martínez Usero
}

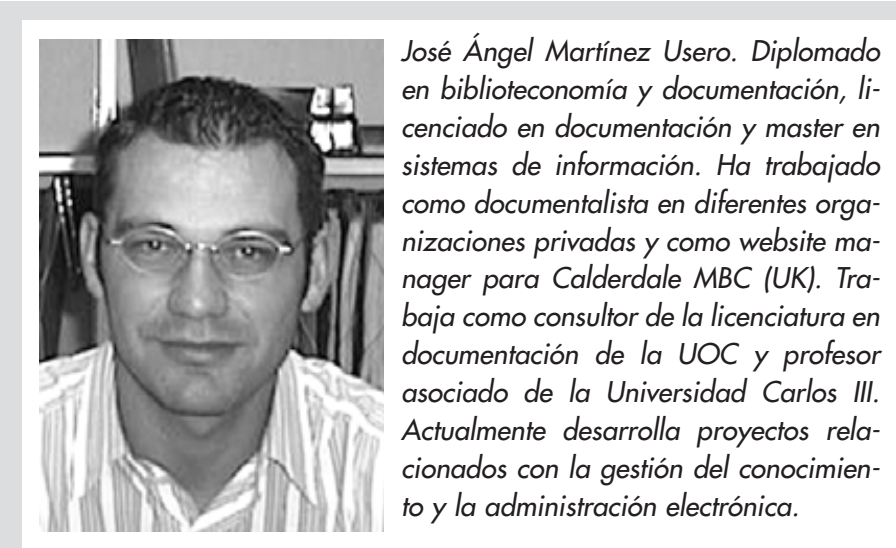

Resumen: Se pone de manifiesto la importancia de las tecnologías del comercio electrónico para el impulso de los servicios característicos de la administración electrónica. Se analizan diversos aspectos tecnológicos y metodológicos en el desarrollo de sitios web corporativos. Se presentan los resultados de diferentes estudios e informes sobre la situación de los sitios web para la oferta de servicios de administración electrónica y se propone un modelo de buenas prácticas basado en los puntos débiles detectados.

Palabras clave: Comercio electrónico, Administración electrónica, Desarrollo web, Sitio web, Tecnología web.

Title: From e-commerce to e-government: technologies and methodologies for information management

Abstract: The importance of e-commerce technologies as the basis for new e-government services is discussed and an analysis of different technological and methodological aspects related to web site development is offered. The results of a series of studies and reports on the current situation of public web sites is presented. Finally, a proposed model of good practice based on the detected weakness in prior research is provided.

Keywords: E-commerce, Electronic commerce, E-government, Electronic government, Web development, Web site, Web technology.

Lara Navarra, Pablo; Martínez Usero, José Ángel. «Del e-commerce al e-government: tecnologías y metodologías para la gestión de información». En: El profesional de la información, 2002, noviembre-diciembre, v. 11, n. 6, pp. 421-435.

Pablo Lara Navarra. Diplomado en biblioteconomía y documentación y licenciado en documentación, es profesor coordinador del área de gestión de la información de la UOC, la Universidad Virtual. Actualmente desarrolla proyectos de visibilidad de información en contenidos educativos online y gestión de recursos electrónicos.

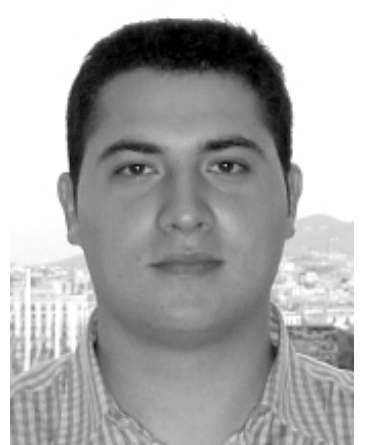

\section{La Administración en la sociedad del conocimiento}

Una encuesta llevada a cabo por The Economist (Sydmonds, 2000) sugiere que tras el comercio electrónico la próxima gran "e-revolution" será la administración electrónica. El informe confirma que las acciones en este ámbito son prioridad en muchos países e incluso han captado la atención de organismos supranacionales como la Unión Europea.

Las denominadas sociedad de la información, nueva economía (e-economy) y sociedad del conocimiento han ocasionado un cambio social real y, de hecho, las transformaciones en el sector privado están provocando fuertes presiones para el lanzamiento de la administración electrónica (Murray, 2001). Si los ciuda- danos pueden comprar online o recibir servicios bancarios, también pueden querer pagar impuestos o solicitar un permiso de aparcamiento online.

La sociedad de la información, ampliamente impulsada por la utilización de las tecnologías de la información y la comunicación (TIC), ha afectado cada vez más al sector público. Las organizaciones públicas siguen el ejemplo del sector privado y aprovechan el enorme potencial de estas tecnologías para aumentar su eficacia mediante la creación de servicios característicos de la administración electrónica.

La nueva economía ha transformado las necesidades de los ciudadanos y las empresas, que requieren servicios de calidad por parte de las organizaciones 


\section{Definición de organización pública:}

La definición de sector público varía de un estado miembro a otro. En la legislación y en los debates sobre el acceso a la información del sector público aparecen 3 enfoques posibles (Comisión Europea, COM, 1998, 585 , p. 12):

-El funcional: según el cual comprende los organismos que ejecutan labores de administración del estado.

-El jurídico-institucional: sólo los organismos cubiertos expresamente por una norma concreta forman parte del sector público.

-El financiero: incluye los organismos financiados principalmente con fondos públicos (es decir, que no actúan siguiendo las leyes habituales del mercado).

Por otra parte, la definición que figura en el artículo I de la Directiva 92/50/CEE del Consejo, de I8 de junio de 1992, sobre coordinación de los procedimientos de adjudicación de contratos públicos de servicios, con arreglo a la cual se entiende por "organismo de derecho público" todo aquel:

- creado específicamente para satisfacer necesidades de interés general, que no tenga carácter industrial o mercantil,

—dotado de personalidad jurídica, y

- cuya actividad esté mayoritariamente financiada por el estado, los entes territoriales u otros organismos de derecho público; o cuya gestión este sujeta a la supervisión de dichos organismos; o tenga un órgano de administración, de dirección o de supervisión, de cuyos miembros más de la mitad sean designados por el estado, los entes territoriales u otros organismos de derecho público.

De esta definición se desprende que para adoptar un carácter integrador con relación a lo que se entiende por "organización pública" debemos tener en cuenta el enfoque financiero, o bien, de mera gestión. En este sentido la mayoría de las unidades de información pueden ser consideradas organizaciones públicas.

públicas. Se ha creado una situación en la que las organizaciones públicas se sienten obligadas a establecer nuevas vías de interacción con los ciudadanos, las empresas y otras organizaciones.

El sector público, por la naturaleza de sus dimensiones y alcance de sus actividades, representa el mayor recurso único de información para crear contenidos y servicios de información de valor añadido ( $C E$ COM, 1998, 585, p. 6). Los resultados de recientes investigaciones indican que entre un $15 \mathrm{y}$ un $25 \%$ del total de datos requeridos en el comercio electrónico se basan en información del sector público (Electronic publishing services, 2000). Se trata de un activo importante con un potencial de crecimiento considerable, que puede convertirse en un factor clave en el ulterior desarrollo del sector de los contenidos (Cornella, 1999). Gloria Nistal ofrece una visión estructurada de las relaciones entre sociedad de la información y administración electrónica en el ámbito internacional, europeo y nacional (tabla 1 ).

\section{Un nuevo concepto: la administración electrónica}

Las organizaciones públicas tienen un importante papel en la nueva economía, facilitando el desarrollo económico y la inclusión social, conceptos que van unidos en esta nueva sociedad. De hecho, sólo las personas con habilidades digitales podrán aprovechar todos los beneficios que la revolución de la información puede proporcionar.

\begin{tabular}{|l|l|l|}
\hline Internacional & Unión Europea & España \\
\hline Information society & eEurope & Info XXI \\
\hline $\begin{array}{l}\text { Movimiento } \\
\text { internacional para la } \\
\text { globalización utilizando } \\
\text { las TIC. }\end{array}$ & $\begin{array}{l}\text { Programas de la UE } \\
\text { para la implantación de } \\
\text { la sociedad de la } \\
\text { información en los } \\
\text { Estados miembros. }\end{array}$ & $\begin{array}{l}\text { Programa del Gobierno español para } \\
\text { la implantación de la sociedad de la } \\
\text { información en España. }\end{array}$ \\
\hline Egovernment & Egovernment & Administración electrónica \\
\hline $\begin{array}{l}\text { En la sociedad de la } \\
\text { información hay un } \\
\text { subconjunto de } \\
\text { actividades y grupos en } \\
\text { el ámbito de las } \\
\text { administraciones } \\
\text { públicas (ONU; } \\
\text { Unesco; Ocde; UE, } \\
\text { etc.) }\end{array}$ & $\begin{array}{l}\text { En el programa de la } \\
\text { UE eEurope, que tiene } \\
10 \text { líneas de acción, la } \\
\text { que se dedica al ámbito } \\
\text { de las administraciones } \\
\text { públicas se denomina } \\
\text { eGovernment. }\end{array}$ & $\begin{array}{l}\text { El programa Info XXI } \text { de tiene } 4 \text { gruposos } \\
\text { administración, para de ellos es el de la la implantación } \\
\text { Españanistración electrónica en }\end{array}$ \\
\hline
\end{tabular}

Tabla 1. Relaciones entre sociedad de la información y administración electrónica. Fuente: Nistal Rosique, EPI, 2002, p. 112. 
Administración electrónica se define como una nueva forma de gestión pública, basada en el uso interactivo de las TIC (internet), con el doble objetivo de la prestación de mejores servicios para ciudadanos y empresas, así como la mejora de los procesos internos de las organizaciones públicas. Ello conlleva la optimización de la gestión interna de las organizaciones y de la prestación de servicios electrónicos.

\section{«Tras el comercio electrónico, la próxima gran 'e-revolution' será la administración electró- nica»}

La administración electrónica implica el uso de internet para el desarrollo de servicios públicos rápidos e interactivos (Liikanen, 2001). Esta acepción incluye 2 aspectos complementarios. Por un lado, los ciudadanos están bien informados y bien atendidos (e-government services). Además pueden participar activamente en los procesos públicos, lo que nos conduce al concepto de democracia electrónica (e-democracy processes).

Los servicios de la administración electrónica abarcan cualquier tipo de servicio, en cualquier momento, 24 horas al día y 7 días a la semana $(24 \times 7)$, desde cualquier lugar y desde multitud de dispositivos de acceso, tales como ordenador, teléfono móvil, televisión digital, PDAs, etc.

La democracia electrónica consiste en un conjunto de aplicaciones de internet que intentan ofrecer canales para la participación de los ciudadanos en la toma de decisiones de las administraciones públicas (Canals, 2001, p. 7).

\begin{tabular}{|l|l|l|}
\hline G2C & Government to citizen & Administración-ciudadano \\
\hline G2B & Government to business & Administración-empresa \\
\hline & & \\
\hline G2G & Government to government & Administración-administración \\
\hline G2E & Government to employee & $\begin{array}{l}\text { Administración-empleados } \\
\text { internos }\end{array}$ \\
\hline
\end{tabular}

Tabla 2. Tipos de interacción en administración electrónica

\begin{tabular}{|l|l|}
\hline Sitio web promocional & $\begin{array}{l}\text { Información básica sobre la organización sin } \\
\text { posibilidad de interacción. }\end{array}$ \\
\hline Portal & $\begin{array}{l}\text { Transacción bidireccional de poca complejidad de } \\
\text { una organización con sus usuarios. }\end{array}$ \\
\hline \multicolumn{2}{|l}{} \\
\hline Ventanilla única & $\begin{array}{l}\text { Integración con la intranet y posibilidad de } \\
\text { gestionar y efectuar pagos online. }\end{array}$ \\
\hline \multicolumn{2}{|l}{} \\
\hline e-Administración & $\begin{array}{l}\text { Integración de todas las organizaciones públicas y } \\
\text { gestión global. }\end{array}$ \\
\hline
\end{tabular}

Tabla 3. Nivel de desarrollo de sitios web para administración electrónica.

\section{Tipos de administración electrónica.}

Todos estos procesos han facilitado la transformación de las relaciones entre los ciudadanos, las empresas y la propia administración. Hoy en día los dos primeros esperan que la administración proporcione servicios y soluciones adecuadas a sus necesidades. Esta exigencia ha generado la aparición en los sitios web de una segmentación de servicios y contenidos por usuarios (Fundación Retevisión, 2002, p. 15).

En el comercio electrónico se identificaron 4 tipos de interacción: B2B (business to business), B2C (business to consumer), B2A (business to administration) y C2A (consumer to administration). De la evolución de los 2 últimos surgen las bases de la administración electrónica. La administración tradicional ha cambiado la gestión interna para responder a las demandas de las empresas (B2A) y los ciudadanos (C2A), dando lugar a nuevas formas de interacción, tal y como podemos observar en la tabla 2 .

\section{Fases de desarrollo de sitios web.}

En los diferentes estudios se propone una clasificación de los sitios web dependiendo de su nivel de sofisticación, calidad de los contenidos o posibilidades de interacción. Una clasificación integradora diferenciaría entre 4 tipos de sitios web para la oferta de servicios de administración electrónica (tabla 3). Teniendo en cuenta nuestra ordenación inicial podemos representar gráficamente cada uno de los niveles de desarrollo con relación a los beneficios que aporta y la complejidad en su avance e implementación. Además, se especifican las características esenciales de cada uno de los posibles niveles de desarrollo (gráfico 1).

3. Los servicios característicos de la administración electrónica.

En marzo de 2001 se aprueba por el Consejo Europeo el listado de 20 servicios públicos básicos para prestar electrónicamente (12 para ciudadanos y 8 para empresas) y que se encuentra clasificado según la naturaleza del servicio (tabla 4).

\section{Del comercio electrónico a la administración electrónica}

La administración electrónica está siguiendo un proceso de implantación relativamente lento en el que influyen, además de la vo- 
luntad política de la UE y el gobierno central, 3 puntos clave:

- La presión social y económica que demanda nuevos servicios y prestaciones por parte de la Administración.

-El desarrollo tecnológico suficiente para la adquisición de tecnología consolidada que permita la implantación de nuevos servicios vía web.

- La tendencia a la incorporación de las TIC en la gestión integral de las organizaciones públicas. (p. ej.: Libro blanco para la mejora de los servicios públicos).

\section{http://www.map.es/libro/pdf.htm}

Todo ello ha provocado un cambio en los procedimientos de gestión interna. En las organizaciones públicas se ha llevado a cabo un proceso de benchmarking (comparación de situación con el entorno) sobre las técnicas de gestión características de las empresas privadas mucho más orientada a obtención de resultados y la gestión del cambio. La tendencia generalizada pasa por la asimilación por parte del sector público de los modelos de comercio electrónico, esto es, de las tecnologías y metodologías de gestión características del comercio electrónico. De esta forma, se están introduciendo nuevos modelos de gestión, tanto para la organización interna (back office) como para el desarrollo de servicios electrónicos del sitio web corporativo (front office)(gráfico 2)

\begin{tabular}{|c|c|}
\hline Recaudatorios & $\begin{array}{l}\text { - Ingreso de impuestos } \\
\text { - Impuestos de sociedades: declaración, presentación } \\
\text { - } \text { Declaraciones de aduanas } \\
\text { - Contribuciones a la Seguridad Social por empleados }\end{array}$ \\
\hline Registrales & $\begin{array}{l}\text { - } \text { Matriculación de coches (nuevos, usados e importados) } \\
\text { - } \text { Certificados (nacimiento, matrimonio, etc.) } \\
\text { - } \text { Registro de nuevas sociedades } \\
\text { - Envío de datos para estadísticas oficiales }\end{array}$ \\
\hline $\begin{array}{c}\text { Facilidades al } \\
\text { ciudadano }\end{array}$ & $\begin{array}{l}\text { - Búsqueda de trabajo a través de las Oficinas de Empleo } \\
\text { - Bibliotecas públicas (disponibilidad de catálogos) } \\
\text { - Senuncias a la Policía } \\
\end{array}$ \\
\hline $\begin{array}{l}\text { Ingresos } \\
\text { económicos }\end{array}$ & $\begin{array}{l}\text { - } \text { Ayudas de la Seguridad Social: subsidio de desempleo } \\
\text { - } \text { Ayuda familiar, gastos médicos (reembolso o pago directo) } \\
\text { - } \text { Compras públicas }\end{array}$ \\
\hline &. \\
\hline $\begin{array}{l}\text { Tramitación } \\
\text { documental, } \\
\text { permisos y } \\
\text { licencias }\end{array}$ & $\begin{array}{l}\text { - } \text { Documentos personales (pasaporte y permiso de conducir) } \\
\text { - } \text { Solicitud de licencias de obra } \\
\text { - Permisos relativos al medioambiente (incluidos informes) }\end{array}$ \\
\hline
\end{tabular}

Tabla 4. Los 20 servicios de la administración electrónica

\section{Beneficios}

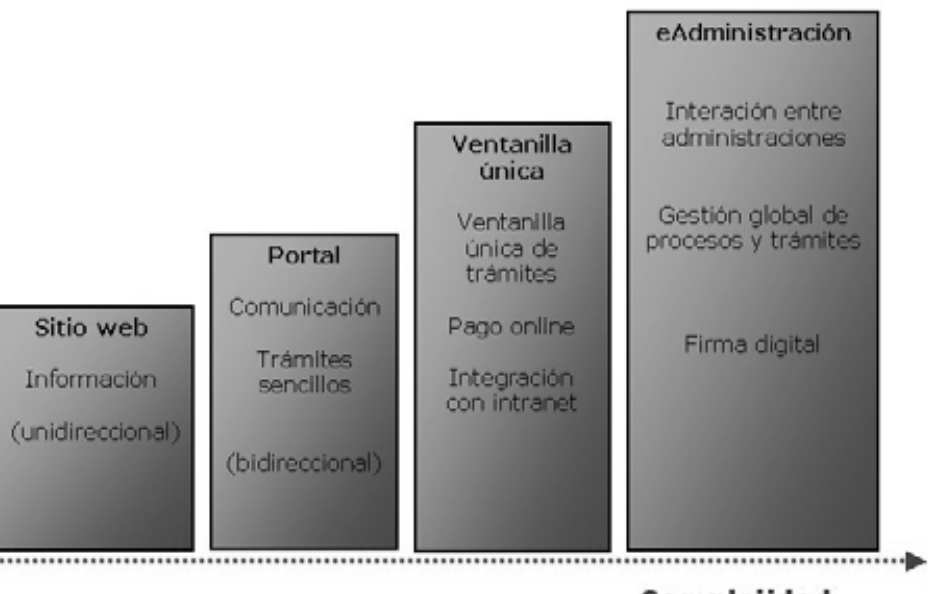

Complejidad

Gráfico 1. Fases de implementación de la administración electrónica. Fuente: Fundación Retevisión, 2002, p. 17

La reorganización y simplificación de la gestión debe actuar como un factor potenciador de nuevos servicios que, de forma funcional, podemos dividir en: servicios públicos electrónicos, democracia electrónica y servicios de información. Finalmente, los conceptos de seguridad, relación con los usuarios, gestión de contenidos, usabilidad y accesibilidad web deben estar presentes en el marco de la estrategia organizacional para la oferta de servicios característicos de la administración electrónica.

\section{La estrategia organizacional (e-strategy)}

El comercio electrónico impulsó el desarrollo del marketing tradicional hacia otro de naturaleza electrónica, las actividades de promoción web y la creación de una estrategia web organizacional. De igual modo, las organizaciones públicas deben plantearse el diseño de una estrategia web con un doble objetivo. Por un lado, satisfacer plenamente las necesidades de los diferentes grupos de usuarios y, por otro, desarrollar servicios y productos de calidad y a un coste mínimo.

Para difundir la existencia de nuestro sitio web, además de las actividades de e-marketing, que son de carácter puntual, se puede llevar a cabo un conjunto de actividades básicas de promoción e incluso un plan de posicionamiento web. 


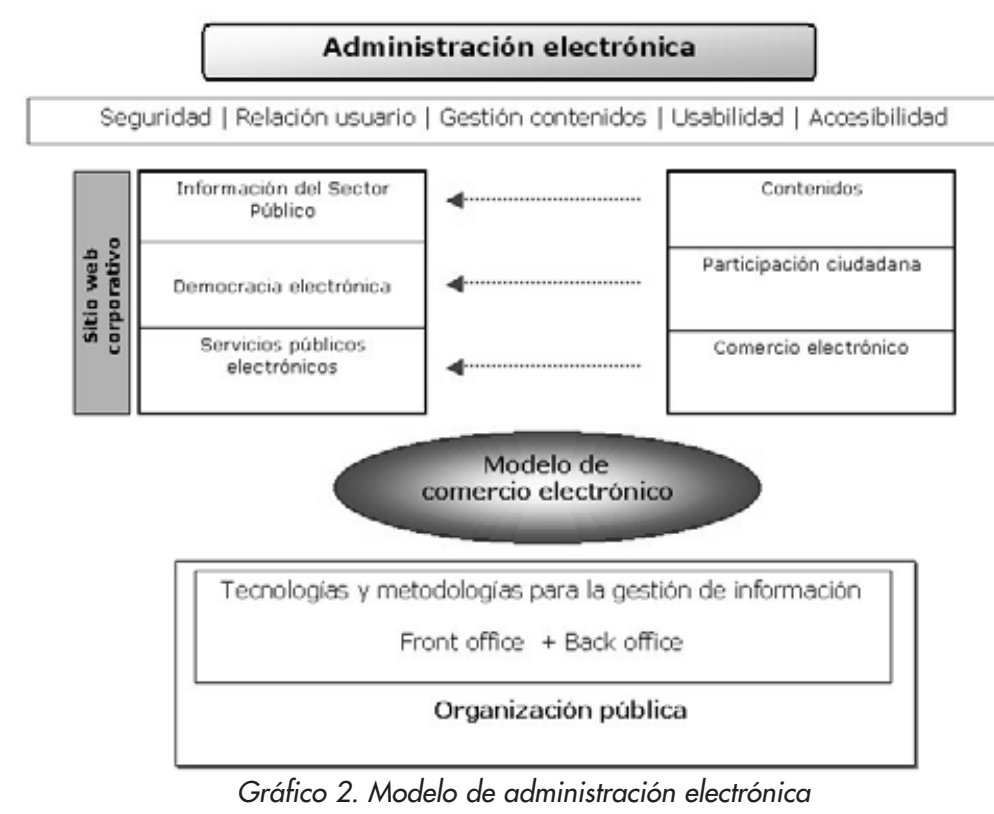

\section{Actividades básicas de promoción.}

- Incorporar la dirección web tanto en la documentación y publicidad de la organización como en la versión impresa de documentos disponibles en la web.

- Intercambiar links con otros sitios web de organizaciones relacionadas.

-Publicitar el sitio web por los medios tradicionales para llegar a nuestra audiencia.

—Darlo a conocer también en otros sitios web clave.

\section{Marketing electrónico.}

La publicidad puede convertirse en una fuente de ingresos en el caso de las organizaciones públicas. Permite reducir el coste de la oferta de servicios vía web, mejorar la calidad de los ya ofrecidos o bien proporcionar una vía más rápida y eficaz para la oferta de servicios online. Actualmente se considera una práctica legítima siempre que esté sometida a ciertas directrices y reglas (Web quality briefings 1, 2001).

El uso de internet con motivos publicitarios o de marketing nos lleva a distinguir 2 tipos de prácticas:

a. Venta de publicidad en nuestro sitio web. No es ésta una tarea fácil, se trata de un área muy competitiva y delicada en la que las organizaciones públicas deben tener especial cuidado en la gestión, venta y promoción de estos servicios. En este sentido es aconsejable dirigirse a consultoras externas (por ej.: COI Communications) que puedan aconsejarnos en cuanto a las reglas del mercado.

http://www.coi.gov.uk

b. Compra de publicidad en otros sitios web. El gobierno del Reino Unido ha publicado un guía (Gui- dance on the work of the government information service, 2001) sobre las buenas prácticas, relación calidad-precio y procedimientos que las organizaciones públicas deben seguir a la hora de contratar cualquier tipo de publicidad, incluyendo la web. Además de tener en consideración estas directrices, como se dijo antes es aconsejable dirigirse a consultoras externas para configurar una estrategia integrada de marketing electrónico para nuestra organización.

\section{Tecnologías y metodologías para la gestión de información web}

Una vez establecida la estrecha relación existente entre comercio y administración electrónica, tanto desde el punto de vista de la gestión interna como de las tecnologías y metodologías para la gestión eficiente de la información, se va a analizar la influencia que el comercio electrónico y sus aplicaciones han tenido en el impulso de los servicios característicos de la administración electrónica.

\section{La seguridad en las transacciones/firma di- gital.}

El comercio electrónico ha sentado los precedentes del pago online y la interacción total del cliente con la empresa. El pago de servicios y productos online es una realidad que ya ha sido transferida desde las aplicaciones de comercio electrónico a los servicios de administración electrónica. Un ejemplo claro lo tenemos en nuestro país, donde la Agencia Tributaria está considerada el organismo fiscal tecnológicamente más desarrollado del mundo.

La introducción de la vía telemática en la gestión tributaria está suponiendo una evolución en dicho sistema, en el sentido de que la Administración, evidentemente sin abandonar su labor de control del cumplimiento de los deberes tributarios por parte de los contribuyentes, potencia su papel asistencial, desarrollando una importante función de información y ayuda al contribuyente, y descargándose de importantes deberes formales (Delgado García, 2002, p. 2).

El reto actual consiste en la identificación de los ciudadanos, no sólo de los datos bancarios y de crédito, sino de la propia persona. El establecimiento de una metodología de identificación unívoca de las personas (e-card) mediante un medio electrónico y seguro se ha convertido en el vacío técnico, metodológico y legal que es necesario cubrir para garantizar los diferentes aspectos que conlleva la e-democracy. 


\section{La relación con el usuario (eCRM).}

La gestión de la relación con las personas se perfila cada vez más como un elemento diferenciador en las organizaciones. En internet, si bien la mayoría de visitas a sitios web las realizan visitantes anónimos de los que no se tiene ningún tipo de información, sí que se puede analizar su comportamiento $\mathrm{y}$, en consecuencia, readaptar y mejorar contenidos y servicios (Berbel; Capillas, 2002).

La gestión de la relación con el cliente (customer relationship management) conlleva la atracción y retención del usuario mediante una estrategia focalizada en él. Una de las claves de la filosofía $C R M$ es el tratamiento de forma única y personalizada de una persona, es decir, debemos conocer todas las acciones realizadas por un cliente potencial, si lo es de la institución y en qué ámbitos. A la vez, tenemos que saber cuáles son sus preferencias, hacerle encuestas de satisfacción, gestionar eficientemente sus quejas, etc. Todo ello, y a pesar de trabajar con grandes volúmenes de clientes, encaminado a un trato personalizado y de calidad.

\section{«En el ámbito del comercio electrónico la usabilidad web ha sido un requisito de super- vivencia; en administración electrónica, la usabilidad es to- davía un lujo»}

El problema en la implementación de estos sistemas reside en la necesidad de grandes inversiones iniciales y el compromiso del área de tecnologías de la información en el desarrollo de herramientas para la atracción, retención y satisfacción del usuario (Jones, 2002, p. 6). Por tanto, sólo las grandes organizaciones pueden abordarlos a corto plazo. Además, en el ámbito de las organizaciones públicas el $C R M$ en los servicios electrónicos se considera un objetivo a largo plazo, no prioritario.

\section{La gestión de contenidos.}

Los materiales característicos en el ámbito del comercio electrónico suelen consistir en datos muy estructurados (bases de datos relacionales sobre productos, clientes, etc.). En cambio, los contenidos habituales de la administración electrónica son muy heterogéneos entre sí y requieren por tanto una gestión especial, generalmente mediante un sistema de gestión de contenidos web (sgcw).

Un sgcw es un conjunto integrado de herramientas para la creación y mantenimiento de bases de datos y todo tipo de información que supone una solución para el crecimiento rápido del sitio web. Puede ser un software construido a medida para este propósito, un sistema más genérico para la gestión del workflow que se utiliza para esta tarea, o bien un grupo de herramientas que en su conjunto pueden ayudar a llevar a cabo la gestión eficiente de los contenidos. Los expertos (Socitm, 2002, p. 61) aconsejan que las sedes web deberían implementar uno a partir de 500 páginas. El sistema ayudará a la organización, de forma genérica, en los siguientes aspectos:

-Agilizar el proceso de creación y publicación de contenidos.

- Permitir a los gestores de información el establecimiento de fecha de revisión/eliminación de los materiales en el momento de su publicación.

- Crear modelos o plantillas (templates) para documentos standard.

- Hacer posible cambios generales en todo el sitio web de forma automática.

- Convertir documentos de un formato a otro (de Word a html, por ejemplo).

-Ofrecer algunas herramientas para el análisis del tráfico en el sitio web.

—Prevenir links rotos.

- Proporcionar enlaces con las bases de datos dinámicas.

El sgcw no va a solucionar los problemas de gestión de contenidos de la organización por sí mismo. El éxito reside en una selección adecuada del producto que se adapte a sus necesidades, así como la correcta implementación y gestión del mismo.

\section{Usabilidad web.}

Consiste en medir la calidad de la relación del usuario cuando interactúa con un producto o sistema, ya sea un sitio web, una aplicación de software, tecnología móvil o cualquier otro sistema de interacción con el usuario. Estos conceptos han tenido gran importancia con el surgimiento del comercio electrónico (Usableweb-ecommerce, 2002), en el que internet se utiliza como un medio de negocio en el cual es necesario atraer, mantener y fidelizar a los clientes.

Los servicios característicos de la administración electrónica se consideran un servicio público de valor añadido; generalmente se ofrece un servicio de información puro o una transacción que implica el intercambio de información. Su estructuración y presentación, así como el diseño del servicio, determinarán la utilidad y eficacia del sitio web. 
Los factores que afectan a la relación del usuario con el producto o sistema son:

-Facilidad de aprendizaje: ¿cómo un usuario que nunca ha visto la interfaz de trabajo anteriormente aprende lo suficiente para llevar a cabo tareas básicas?

-Eficiencia de uso: una vez que un usuario ha aprendido a utilizarlo, ¿con qué rapidez es capaz de realizar diferentes tareas?

- Capacidad de memorización: si un usuario ha usado el sistema anteriormente, ¿le es posible recordar lo suficiente para utilizarlo de una forma eficiente la próxima vez, o tiene que aprender todo de nuevo?

-Frecuencia e importancia de los errores: ¿con qué periodicidad el usuario comete fallos mientras utiliza el sistema, qué importancia tienen estos y cómo los solventa?

\section{«En los sitios web para activi- dades de comercio electrónico, los aspectos de accesibilidad web son una funcionalidad añadida y pocas veces tenida en cuenta. Al contrario, en los sitios web de organizaciones públicas, la accesibilidad web es un requisito»}

- Satisfacción personal: ¿en qué medida le gusta al usuario interactuar con el sistema?

a. Importancia de la usabilidad. Según se informa en las páginas que el Nat. Cancer Institute, EUA, dedica a este tema, las investigaciones llevadas a cabo por User Interface Engineering, Inc., muestran que los usuarios no son capaces de encontrar la información que buscan en la web en un $60 \%$ de las ocasiones. Esto puede conducir a una pérdida de tiempo, reducción de la productividad, incremento de la frustración y a la decisión de no volver a visitar ese sitio web.

\section{http://usability.gov/basics/}

Jakob Nielsen comenta (Jakob Nielsen's alertbox, 28-11-99): "Los estudios sobre la conducta de usuarios en la web muestran una baja tolerancia hacia los sitos con dificultades de diseño o extremadamente lentos. Los usuarios no esperan y no quieren aprender cómo usar un sitio web, al contrario, deben ser capaces de intuir el funcionamiento del sitio web inmediatamente después de un primer vistazo a la página principal”.

\section{http://www.useit.com}

b. Metodología de la usabilidad. No existe una en concreto que pueda aplicarse a todos los sistemas. Sin embargo, en todo programa de usabilidad se pueden identificar 6 fases bien diferenciadas:

b.1. Planificación del sitio web. El primer paso consiste en entender: ¿por qué se está creando?, ¿quiénes son los usuarios potenciales?, ¿cuándo y por qué los usuarios lo visitan? Respondiendo a estas preguntas se obtienen los objetivos generales del sitio web ya que los específicos van a depender del tipo de organización que ofrece servicios y de la naturaleza de los usuarios que los requieren.

b.2. Recogida de datos de los usuarios. Dado que el diseño web se basa en las necesidades de éstos, debe ser recogido un conjunto de datos sobre ellas, y cómo el sitio web existente (si hay uno) responde a tales necesidades. Existen diferentes métodos para la obtención de dichos datos, entre ellos: formularios de opinión, sistemas métricos (Jacob Nielsen's alerbox, 2101-01) y test de usabilidad del sitio web existente.

b.3. Desarrollo de un prototipo. Para un usuario es más sencillo reaccionar ante un ejemplo de sitio web que teorizar sobre lo que funcionaría mejor. Por tanto, la construcción de un prototipo puede ofrecer resultados muy útiles y puede ser usado para obtener los comentarios de los usuarios y observar la habilidad del modelo para satisfacer las necesidades básicas de quienes lo usan (Jacob Nielsen's alertbox, 05-08-01).

b.4. Recogida, desarrollo y revisión de contenidos. Los materiales deben estar estructurados de forma que respondan de una forma eficiente a las necesidades de los usuarios, una buena estructura de la información y unos contenidos claros y específicos son esenciales para que se pueda localizar lo que se necesita de una forma rápida y eficaz.

b.5. Implementación de tests de usabilidad. Con estas herramientas se trata de averiguar cómo ayudar a los usuarios a realizar las tareas que necesitan y qué puede impedirles llevarlas a cabo. A partir del prototipo se implementan acciones que el usuario deberá hacer. Entonces se recogen datos detallados sobre sus éxitos y fracasos en la resolución de las tareas planteadas y, a partir de ahí, tenemos una base de conocimiento que nos va a permitir modificar ciertos aspectos del prototipo inicial.

b.6. Evaluación continua del sitio web. Una vez haya sido implementado, los responsables de su mantenimiento deben recopilar información que permita evaluar la respuesta del usuario ante los diferentes aspectos de usabilidad que hemos intentado desarrollar en él.

c. Campo de aplicación. Los tests de usabilidad se aplican a diferentes áreas, dependiendo de la tipología de producto, la tecnología utilizada, los usuarios fina- 
les, etc., y existen por tanto test concretos para cada una de estas áreas, por ejemplo:

-Aplicaciones de tecnología web: internet, intranet, WAP, etc.

-Áreas específicas de una aplicación web: home page, motor de búsqueda interno, site index, etc.

-Iniciativas electrónicas: e-commerce, e-learning, e-government, etc.

- Segmentos de audiencia: usuarios con discapacidad, de edad avanzada, niños, etc.

\section{Accesibilidad web.}

Se trata de un nuevo concepto que va más allá de la usabilidad, pero que puede ser considerado como una parte o variante de ella. De hecho, según los expertos (Eisenstadt, 2000), si tenemos en cuenta las necesidades de los usuarios con algún tipo de discapacidad a la hora de desarrollar un sitio web, el conjunto de usuarios, discapacitados o no, se beneficiará y ello aumentará la usabilidad global. Tradicionalmente se ha considerado que el desarrollo de sitios web accesibles conlleva un coste añadido, su diseño no es atractivo y sus contenidos son escasos. Pero la tecnología ha avanzado hasta tal punto que se puede conseguir la accesibilidad e incorporar a su vez las más avanzadas técnicas de desarrollo web.

El enfoque de la Unión Europea para asegurar la accesibilidad de los sitios web de carácter público está recogido en el plan de acción e-Europe 2002: una sociedad de la información para todos. En el objetivo 2c se incluyen 5 puntos a conseguir, bajo el nombre genérico de e-accessibility para la promoción de la participación de todos los ciudadanos en una sociedad basada en el conocimiento (COM 2001, 607 final). Su sucesor, e-Europe 2005 (COM 2002, 265 final) continúa la labor emprendida por e-Europe 2002.

\section{«El pago de servicios y produc- tos online es una realidad que ya ha sido transferida desde las aplicaciones de comercio electrónico a los servicios de administración electrónica»}

El 25 de septiembre de 2001 la Comisión adoptó una comunicación (e-accessibiliy, 2002) para mejorar la accesibilidad de los sitios web de carácter público y sus contenidos. El principal objetivo consiste en hacerlos más accesibles para que las personas con algún tipo de discapacidad y/o de edad avanzada puedan acceder a la información y disfrutar de todo el potencial de la administración electrónica.

\begin{tabular}{|c|c|c|}
\hline Prioridad 1 & Adecuación de nivel A & W3C $\begin{array}{r}\text { WAI-A } \\
\text { WCAG } 1.0\end{array}$ \\
\hline Prioridad 2 & Adecuación de nivel AA & WBC WAI-AA \\
\hline Prioridad 3 & Adecuación de nivel AAA & WBC WAI-AAA \\
\hline
\end{tabular}

Las acciones para realizar esta mejora serán ejecutadas por las instituciones europeas y los 15 estados miembros de la Unión Europea mediante la adopción de las directrices WAI (Web Accessibility Initiative), que establecen 3 niveles de accesibilidad (A, AA y AAA) dependiendo del grado de conformidad con las prioridades 1,2 y 3 de las directrices.

http://www.w3c.org/WAI/

Las directrices WAI establecen tres niveles de accesibilidad (A, AA y AAA) dependiendo del grado de conformidad con las prioridades 1,2 y 3 de las directrices (tabla 5).

-Prioridad 1 (Nivel A). El desarrollador de contenidos web debe satisfacer estos puntos, de lo contrario, uno o más grupos de usuarios tendrá dificultades para acceder a la información. La consecución de estos puntos es un requerimiento básico para que algunos grupos de usuarios sean capaces de usar documentos web. El sitio web puede ser certificado con el logo de nivel A.

-Prioridad 2 (Nivel AA). El desarrollador de contenidos web debería satisfacer estos puntos, de lo contrario, uno o más grupos de usuarios tendrá dificultades para acceder a la información. La consecución de estos puntos reduce barreras significantes para el acceso a documentos web. El sitio web puede ser certificado con el logo de nivel AA.

-Prioridad 3 (Nivel AAA). El desarrollador de contenidos web puede satisfacer estos puntos, de lo contrario, uno o más grupos de usuarios tendrá dificultades para acceder a la información. La consecución de estos puntos mejorará el acceso a los documentos web. El sitio web puede ser certificado con el logo de nivel AAA.

Debemos tener en cuenta que W3C WAI no es una norma, sino un conjunto de directrices y, por tanto, no existe una vía automática para validar un sitio web con relación a ellas, sino una prueba de adecuación que permite la certificación y señalar el sitio con un logo.

\section{Herramientas de análisis.}

-Bobby Approval. El mejor programa para analizar la accesibilidad de un sitio web y recibir información sobre los aspectos que no cumple es Bobby, ela- 
borado por The Center for Applied Special Technology (Cast) y adquirido en junio de 2002 por Watchfire.

http://www.cast.org/

http://bobby.watchfire.com/bobby/html/en/index.jsp

- TAW (Test de Accesibilida Web). Es una herramienta en castellano similar a Bobby que permite conocer los problemas de accesibilidad que presenta un sitio web para cada uno de los niveles de accesibilidad.

http://www.tawdis.net

\section{Desarrollo de la administración electrónica en España}

La iniciativa Info XXI: la sociedad de la inform@ción para todos, representa una apuesta clara y decidida para construir la sociedad de la información en España. Consiste en un conjunto estructurado de programas y medidas de actuación que contribuyan a impulsar eficazmente desde el Gobierno el desarrollo integral de la sociedad de la información en nuestro país y su acercamiento a la sociedad civil y a los ciudadanos.

\section{http://www.map.es/csi/sociedadinformacion.htm}

Existe una opinión unánime sobre la importancia de que las administraciones públicas incorporen internamente el uso de las TIC, como catalizador de nuevos desarrollos y con un gran efecto de demostración y arrastre tanto para los ciudadanos como para todos los demás segmentos de la sociedad.

Para analizar la situación actual en nuestro país ofrecemos la información y conclusiones recogidas en diferentes estudios e informes realizados por diferentes organizaciones y particulares con el objetivo de realizar una radiografía de la situación de los sitios web de organizaciones públicas y los servicios característicos de la administración electrónica.

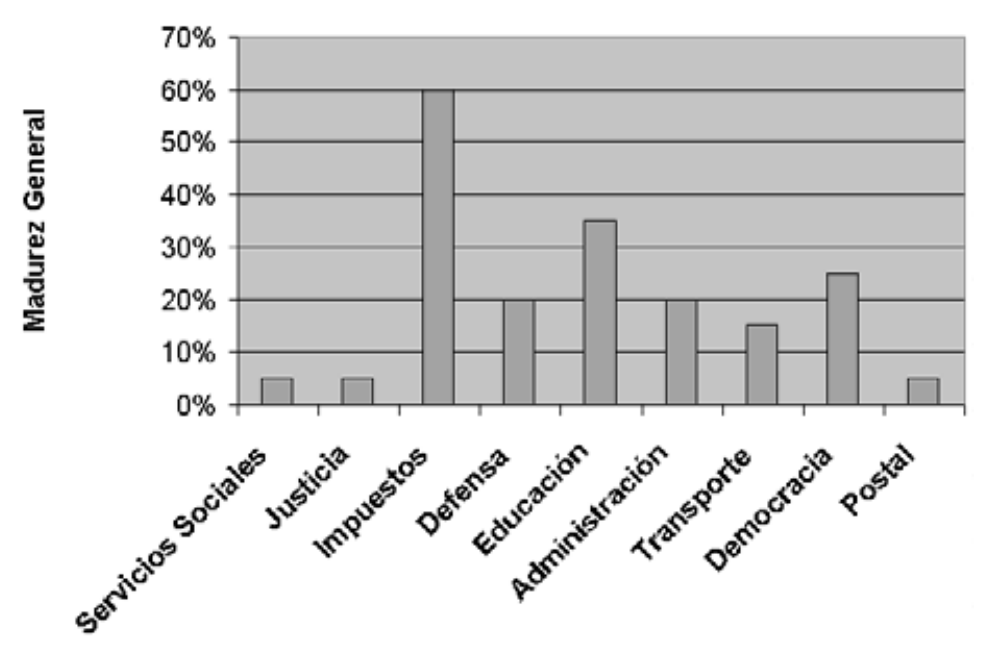

Gráfico 3. Madurez de los servicios por sectores. Fuente: Accenture, 2001, p. 55.
1. La madurez de los servicios de la administración electrónica.

El estudio de Accenture (Acccenture, 2001, pp. 5455) comenta que a pesar de los esfuerzos del gobierno español con la iniciativa Info XXI no se ha conseguido una visión uniforme en materia de administración electrónica. Los resultados de la investigación muestran que no se está alcanzando el nivel de madurez esperado. El gobierno central es responsable de 115 servicios evaluados, de los cuales 62 (el 54\%) se ofrecen de alguna manera a través de internet. En cuanto a su grado de sofisticación, 46 de ellos son meramente sitios web promocionales, 9 pueden ser considerados portales y los 7 restantes permiten transacciones a través de la Red, lo que los convierte en ventanillas únicas.

\section{«Según Accenture, a pesar de los esfuerzos del gobierno es- pañol, con Info XXI no se ha conseguido una visión unifor- me en materia de administra- ción electrónica»}

Un análisis más profundo nos indica que 5 de los 7 servicios considerados como ventanillas únicas están ligados a las actividades de impuestos. Como muestra el gráfico 3 respecto a la comparación del nivel de madurez de los servicios públicos online, fuera del sector de los impuestos el potencial es muy escaso.

\section{La implementación de los $\mathbf{5}$ portales básicos.}

El informe Public e-services within the European Union today, 2001, cuyas principales conclusiones vienen cifradas en el Informe sobre la evolución de los servicios públicos electrónicos dentro de la Unión Europea (Fundación Retevisión, 2002, pp. 14-20), analiza la evolución en cada país de la denominada primera fase de servicios electrónicos. Se analizan los siguientes servicios:

—Existencia de un portal único de acceso a las administraciones públicas.

—Boletín Oficial del Estado disponible en línea.

-Existencia de un portal de búsqueda de la legislación nacional y comunitaria.

- Guía electrónica para el ciudadano explicando los principales procedimientos administrativos.

-Existencia de un portal específico para empresas.

En los resultados España aparece entre los 6 países (junto con Dinamarca, Suecia, Alemania, 
Austria y Reino Unido) que ya han implementado los 5 servicios, aunque con algunos matices.

Después del boom de apariciones de sitios web de las diferentes administraciones (la necesidad de estar presentes en internet y poder así interactuar con

\begin{tabular}{|c|c|l|}
\hline Fase 1 & Información & Información en línea sobre el servicio público \\
\hline \multicolumn{3}{|c|}{} \\
\hline Fase 2 & Interacción & Descarga en línea de formularios \\
\hline Fase 3 & $\begin{array}{c}\text { Interacción } \\
\text { bilateral }\end{array}$ & $\begin{array}{l}\text { Permite la cumplimentación y envío de } \\
\text { formularios, el acceso a bases de datos, etc. }\end{array}$ \\
\hline \multicolumn{3}{|c|}{} \\
\hline \multicolumn{3}{|c|}{ Fase 4 } \\
\hline
\end{tabular}
Tabla 6 sus usuarios), ahora estamos en el momento de la creación de los portales (ventanilla única) que facilitan la navegación por las diferentes administraciones (local, regional y estatal) evitando entrar en todas ellas.

La integración total no se ha conseguido, aunque la mayoría de portales (Reino Unido, España, Dinamarca, Suecia y Francia) que ofrecen transacciones de competencia local y regional, éstas se hacen a través de links (a la página web del ayuntamiento correspondiente, o al gobierno regional) y no se encuentran integradas en el portal (diferente diseño de páginas, de navegación, de mecanismos de interacción, etc.). Son "portales-bases de datos" que centralizan la información dispersa por varios departamentos.

En España, al igual que sucede en otros países, hay alguna comunidad autónoma que se está planteando crear su portal de administración abierta que integre los 3 niveles (local, regional y estatal). Este es el caso de Cataluña que posee un portal propio.

\section{Los portales únicos.}

La Fundación Retevisión ha realizado un estudio comparativo de 6 portales europeos, e-España 2002: situación de España ante la sociedad de la información (Fundación Retevisión, 2002). En él, se analizan con criterios cualitativos los 6 portales siguientes:

-Dinamarca.

๑Espańa $\square$ UE

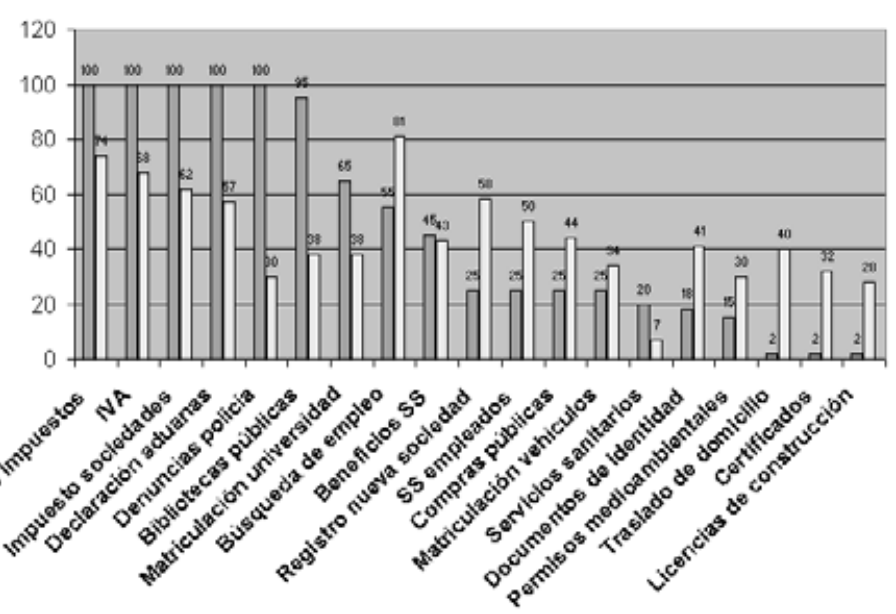

Gráfico 4. Los indicadores de la e-administración España/UE. Fuente: Cap Gemini Ernst \& Young, octubre de 2001. http://www.danmark.dk

-España.

http://www.administracion.es

-Francia.

http://www.service-public.fr

-Italia.

http://www.paforum.net

-Reino Unido.

http://www.ukonline.gov.uk

-Suecia.

http://www.statskontoret.selegov

Los criterios de análisis utilizados han sido los ámbitos y niveles de:

-Información.

—Usabilidad.

—Participación ciudadana.

-Interacción y transacción.

-Fidelización.

Las conclusiones para España destacan que, gracias a la Agencia Tributaria, nuestro país se encuentra entre la fase de portal y la de ventanilla única, pero aún está lejos de poder ser considerada una administración electrónica desarrollada e integrada.

\section{Grado de implantación de los servicios de la administración electrónica.}

Para analizar cuál es la situación actual recurrimos a la primera encuesta sobre servicios públicos electrónicos realizada por Cap Gemini Ernst \& Young en octubre de 2001 (Cap Gemini Ernst \& Young, 2001), que mide el grado en el que los diferentes países están implementando la lista de los 20 indicadores. El universo lo componen los 15 países miembros de la Unión Europea, más Noruega e Islandia. Para la medición del grado de avance en estos indicadores se fijan 4 etapas o fases, dependiendo del grado de desarrollo electrónico del servicio. Las 4 fases son las que podemos ver en la tabla 6. Los resultados se expresan en \%, 
que expresa el grado de implantación o/y sofisticación del servicio, correspondiendo el $100 \%$ a la completa implantación de la fase 4 .

A través del gráfico 4 podemos ver la situación de nuestro país en comparación con la media de los 15 países analizados.

\section{Las mejores prácticas en España.}

En el anexo del informe sobre la evolución de los servicios públicos electrónicos dentro de la Unión Europea (Fundación Retevisión, 2002, p. 70) se enuncian las mejores prácticas en cada uno de los estados miembros. Para el caso de España tenemos:

- Agencia Española de Administración Tributaria. http://www.aeat.es

\section{Interior).}

\section{—Dirección General de la Policía (Ministerio del} http://www.policia.es

\section{- Generalitat de Cataluña.}

$\square N^{\circ}$ de servicios

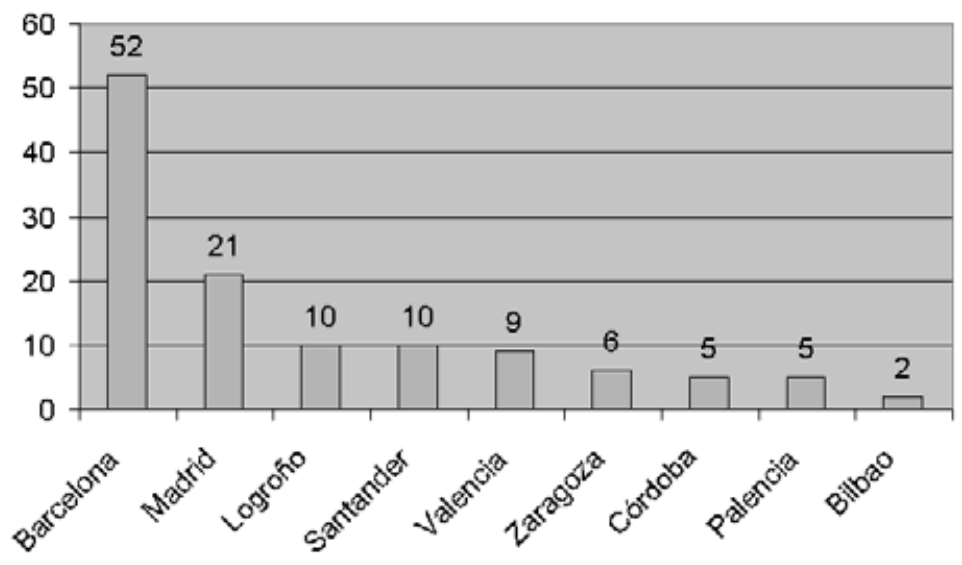

Gráfico 6. Trámites en red. Fuente: Ciberp@is, 2001

\section{$\mathrm{N}^{\circ}$ de impresos}

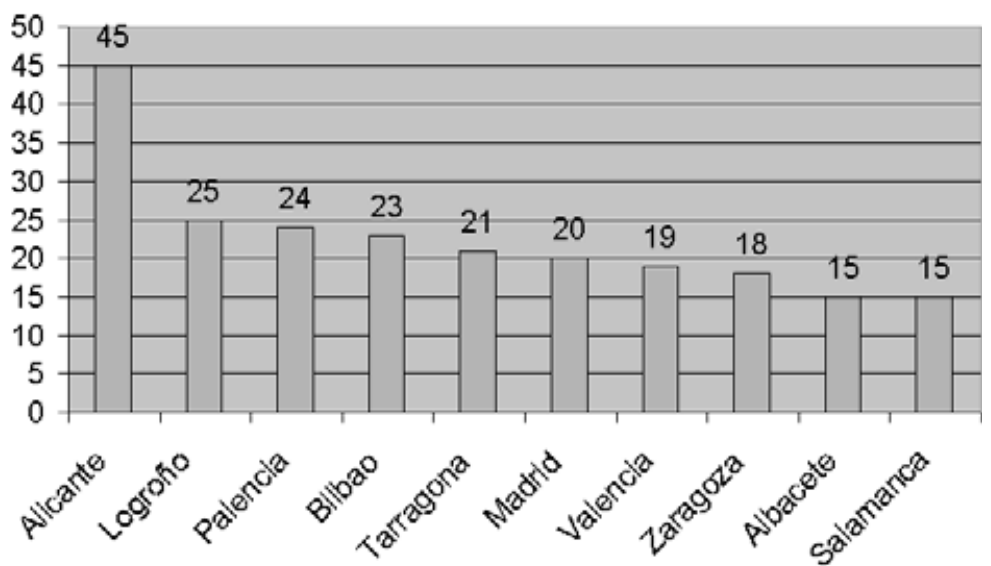

Gráfico 7. Descarga de impresos. Fuente: Ciberp@is, 2001 $\square$ España $\square$ Media Nacional UE

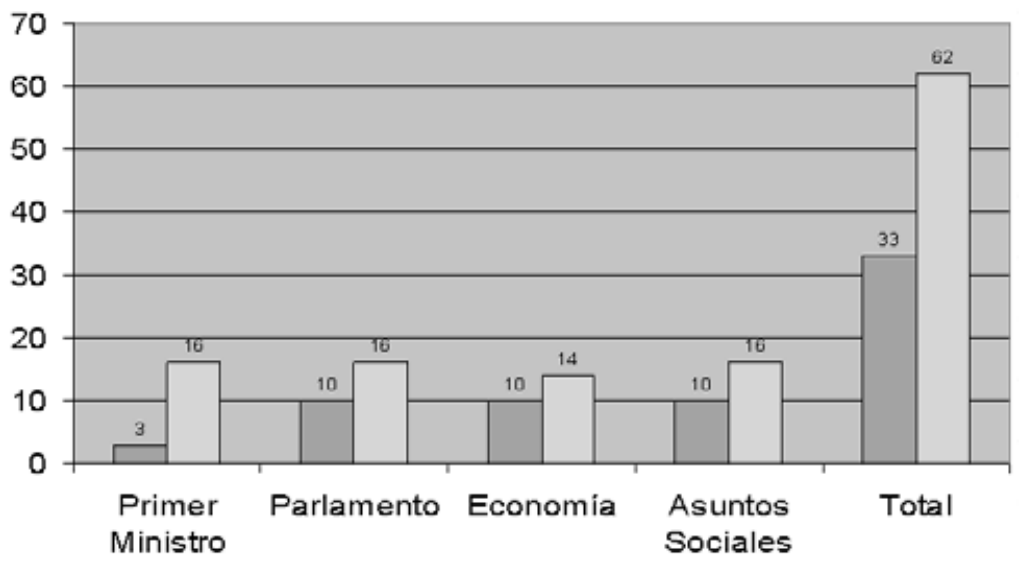

http://www.edu365.com

- Generalitat de Valencia.

http://www.moderniza.com

\section{Las malas prácticas en España.}

La encuesta de Internet Intelligence 2001 (Politics Online, 2001) que evalúa la calidad de los sitios web gubernamentales en los 15 estados miembros, destaca la mala situación de España. Nuestro país está a la cola en las 4 categorías de sitios web analizados (del primer ministro, del parlamento, de economía y de asuntos sociales). El gráfico 5 muestra los resultados de España con relación a la media de la Unión Europea alcanzada en esta encuesta. Los datos fueron recogidos en un proyecto de investigación de la Universidad de Verano de Amsterdam-Maastricht. Cada sitio web gubernamental fue evaluado según 4 criterios específicos, con una puntuación máxima de 25 en cada categoría. La máxima puntuación total, suma de las 4 categorías, es 100.

\section{Radiografía de los sitios web de las 52 ca- pitales españolas.}

La redacción deCiberp@ís llevó a cabo un estudio de los sitios web de los ayuntamientos de las 52 capitales españolas (Ciberp@ís, 2001), del cual se desprendía que 6 de los 52 casos analizados todavía no disponían de sitio web o se encontraba inactivo. Además, se clasifican los servicios ofrecidos, dándoles una puntuación en relación con su utilidad para los ciudadanos. Los más ponderados son los trámites en red y la descarga de impresos, seguidos de pagos en red, callejero, información de transporte, datos del tráfico en directo e idiomas. Los resultados para los 2 primeros servicios se ofrecen en los gráficos 6 y 7. De ellos se desprende la escasa interactividad con el 
ciudadano. Los ayuntamientos deben trabajar duro para ofrecer más recursos electrónicos y fomentar las transacciones vía web. De hecho, el gobierno del Reino Unido tiene como meta ofrecerlos todos de forma electrónica para 2005.

De forma genérica podemos decir que la navegabilidad, esto es, facilidad y claridad de la página, es la asignatura pendiente. Otro aspecto importante está relacionado con la visibilidad, en cuanto que el estudio señala la imposibilidad de adivinar el url de un ayuntamiento español. No existe criterio alguno, a diferencia de otros países, como Italia o Reino Unido donde la dirección URL de los ayuntamientos está normalizada.

8. Estructura de la información en los sitios web de las comunidades autónomas.

Vianello Osti y Sánchez Blanco llevaron a cabo durante marzo de 2000 un análisis de los sitios web de las comunidades autónomas españolas (Vianello, 2000). Se centraron en 2 aspectos: el análisis formal y de contenido de los sitios web. Los resultados se muestran en los gráficos 8 y 9. Las conclusiones genéricas se pueden resumir en los puntos siguientes:

- Se pone de manifiesto que la información debe ser organizada de forma clara y dirigir al usuario hacia algo previsible.

- Se evidencia la necesidad por parte de estas administraciones de operar una transición desde la óptica de productores a una perspectiva de servicio, basada en la actuación cooperativa que garantice junto con la actualidad y la vigencia de la información, la vitalidad que sólo la participación permite.

- Se destaca la importancia de la participación de los profesionales de la información en el desarrollo de sitios web con el objeto de que la información se estructure correctamente y sea verdaderamente accesible al usuario (usable).

\section{Información parlamentaria autonómica en internet.}

Marcos y Gil realizaron un análisis de la información que ofrecen los sitios web parlamentarios de las comunidades autónomas españolas a finales del año 2001, tomando como punto de partida las pautas aprobadas por el Consejo Interparlamentario en su sesión de Amman en el año 2000 y adaptándolas a las peculiaridades de las autonomías españolas (Marcos, 2002). El estudio se centró en 3 aspectos:
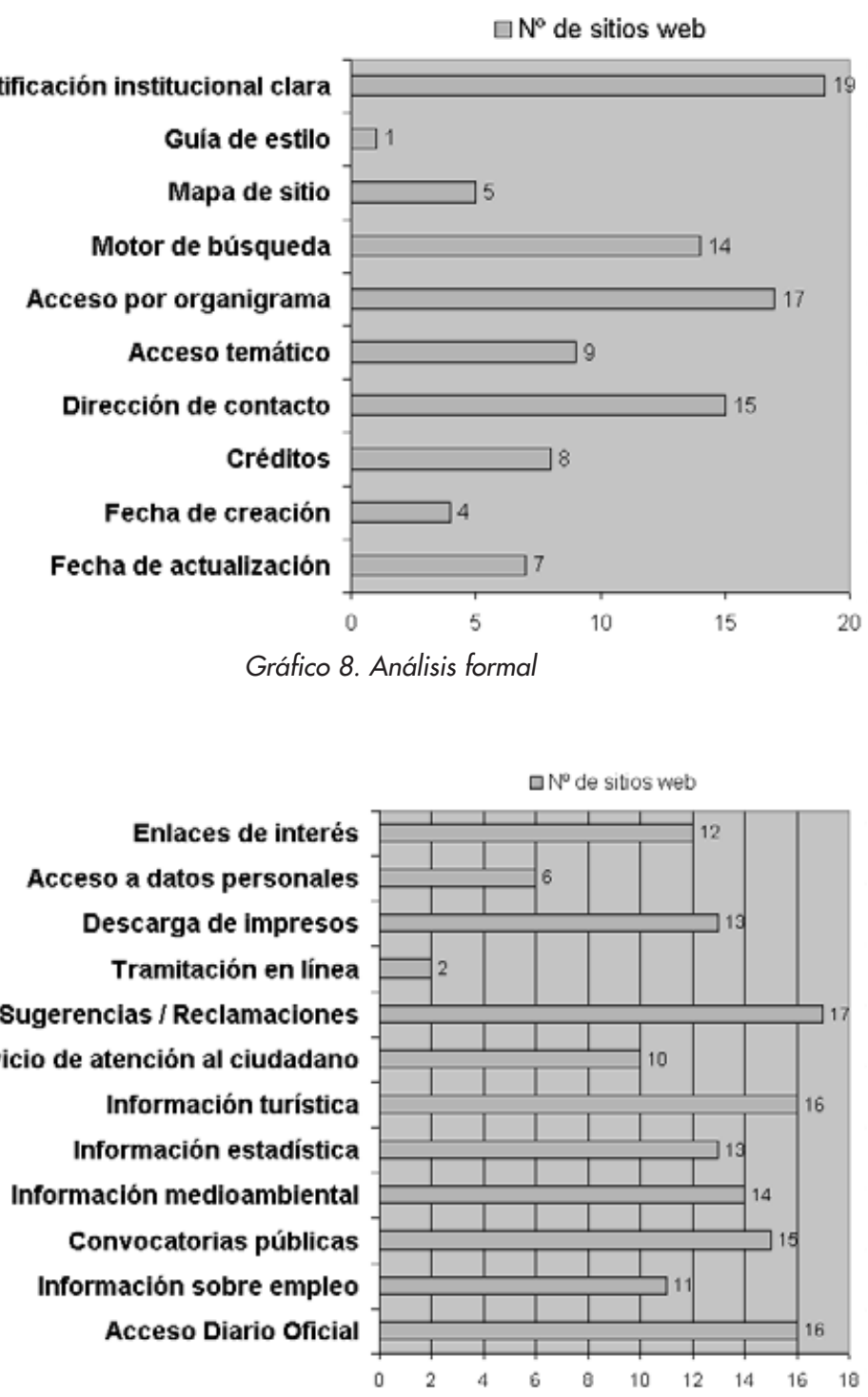

Gráfico 9. Análisis de contenido

-Información que se ofrece sobre la propia institución.

-Elementos que fomentan la participación ciudadana en la vida parlamentaria.

- Herramientas que favorecen la accesibilidad a la información.

Los resultados obtenidos son presentados en el gráfico 10. Del análisis de los datos se concluye que:

- Los parlamentos aportan una gran variedad de datos en todos los aspectos analizados.

— La interacción del ciudadano con los órganos y miembros de la cámara queda cubierta con la provisión de correo electrónico, buzones de participación y foros de discusión.

—El acceso a los contenidos debe ser asistido con herramientas como: mapa de la web y buscador interno desde la página principal. 


\section{Modelo de buenas prácticas}

Tras el estudio llevado a cabo sobre los aspectos esenciales en el desarrollo de sitios web para administración electrónica y la influencia de los modelos de comercio electrónico en la consolidación de tecnologías y metodologías para la gestión de información, proponemos un modelo basado en 10 aspectos clave que las organizaciones públicas deben tener en cuenta como buenas prácticas en el desarrollo de sitios web para la oferta de servicios característicos de la administración electrónica (tabla 7).

1. El sitio web corporativo debe abandonar su carácter esencialmente organizacional para convertirse en un portal para la comunidad a la que sirve, presentado los contenidos y servicios de la forma en la que los usuarios desean recibirlos.

2. La gestión eficiente de los contenidos pasa por la integración de los datos en un sistema de información para el conjunto de la organización, por ejemplo mediante un sistema gestor de contenidos web.

3. La actualización de los contenidos debe ser dinámica y descentralizada, en la que diferentes áreas o departamentos de la organización colaboran.

4. El sitio web no tiene que ser estructurado de una forma departamental siguiendo la estructura de la organización, los usuarios no tienen por qué conocerla para ser capaces de utilizar el sitio web de una forma eficiente.

5. Los contenidos deben presentarse de una forma interactiva cuando sea posible, se debe potenciar la interacción con el usuario y la realización de todo tipo de transacciones online. $\square$ Accesibilidad $\square$ Participación ciudadana $\square$ Elementos informativos

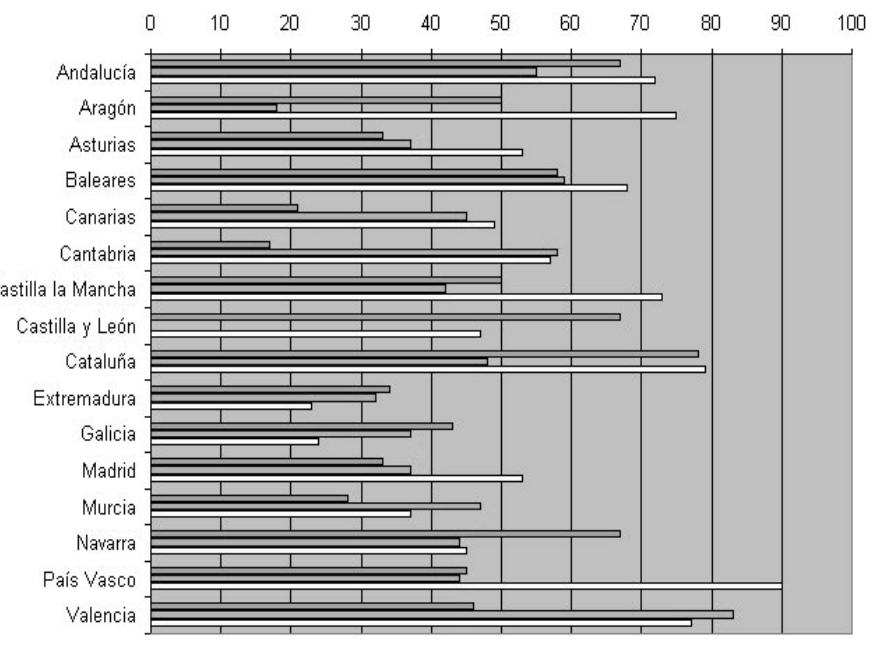

Gráfico 10. Información parlamentaria por autonomías

6. El diseño debe ser atractivo para el usuario, la organización de los contenidos clara y dirigir al usuario hacia algo previsible.

7. El sitio web corporativo y la intranet de la organización deben funcionar de forma integrada con el objetivo de no repetir esfuerzos; por tanto es necesario establecer unos criterios para decidir la información que se publica en cada uno de ellos.

8. Debe ser intuitivo y fácil de usar. Para ello hay que realizar tests y evaluaciones de usabilidad web.

9. Hay que asegurar que los contenidos van a ser accesibles para los usuarios con algún tipo de discapacidad. Los sitios web de las organizaciones públicas tienen la obligación de cumplir al menos la prioridad 1 de WAI.

10. Su gestión no debe estar centralizada en un webmaster o equipo web, sino distribuida en el conjunto de la organización, fomentando asimismo la colaboración entre departamentos.

\section{Conclusiones}

Las tecnologías y metodologías de comercio electrónico han sido la base para el desarrollo de los servicios característicos de la administración electrónica, la creación de nuevas necesidades sociales relacionadas con las tecnologías de la información y la consolidación de lo que podríamos llamar una "cultura informacional". La característica esencial que diferencia los servicios de administración electrónica de 
aquellos de comercio electrónico consiste en la tipología de usuarios/clientes de la primera, en la que todos los ciudadanos, las empresas y otras organizaciones públicas pueden acceder a los ofrecidos vía web, y la Administración tiene asimismo la obligación de implementarlos.

\section{"Gracias a la Agencia Tributa- ria, considerada el organismo fiscal tecnológicamente más desarrollado del mundo, nues- tro país se encuentra entre la fase de portal y la de ventanilla única»}

Las tecnologías y metodologías utilizadas para el desarrollo de los servicios de administración electrónica incluyen nuevos aspectos diferenciadores respecto del comercio electrónico, tales como: la necesidad de métodos de identificación unívoca de las personas (p. ej., procesos electorales, $e$-voting), las gestión de todo tipo de contenidos y transacciones (p. ej., para licitaciones y suministros, e-procurement) así como la implementación de normas de accesibilidad universal ( $e$ accessibility). Para las empresas, la información y los servicios son una forma de negocio, pero para las organizaciones públicas constituyen una obligación para/con la sociedad del conocimiento.

Los estudios dedicados a analizar la evolución y situación actual de los servicios de la administración electrónica ofrecen resultados muy diferentes según el punto de vista del análisis. La situación en nuestro país varía considerablemente dependiendo de si se lleva a cabo teniendo en cuenta aspectos cuantitativos o cualitativos, o bien qué sector de las organizaciones públicas se examine.

Actualmente estamos bastante lejos de conseguir los objetivos marcados en el desarrollo de servicios característicos de la administración electrónica en nuestro país. De hecho, desde el Gobierno central se ofrecen las directrices políticas pero no unas recomendaciones técnicas y metodológicas para el desarrollo de sitios web y servicios en este nuevo ámbito, una acción centralizada ayudaría al conjunto de las organizaciones públicas a implementar servicios con unas garantías mínimas de calidad y con un criterio uniforme para todas las que potencialmente pueden ofrecer servicios de administración electrónica.

\section{Bibliografía}

Berbel, Genís; Capillas, Ramón. Gestión de la relación con las personas en la web: VRM y CRM, portal UOC, 2002.

http://www.uoc.edu/web/esp/art/uoc/berbel_capillas0302/berbel_capillasO 302.html

\section{Recursos seleccionados}

\section{Políticas europeas y nacionales}

-Iniciativa e-Europe. http://europa.eu.int/information_society/eeurope/ index_en.htm

-Iniciativa Info XXI. http://www.map.es/csi/sociedadinformacion.htm

—UK Government guidelines. http://www.e-envoy.gov.uk/webguidelines.htm

\section{Usabilidad web}

—Jackob Nielsen's website http://www.useit.com

-Usable.Net

http://www.usablenet.com

—Usableweb.com

http://www.usableweb.com

\section{Accesibilidad web}

-Web accessibility initiative. http://www.w3c.org/WAl/

-Royal Nat. Inst. of the Blind, UK (Rnib). http://www.rnib.co.uk/access/welcome.htm http://www.rnib.org.uk/digital/welcome.htm

—Fundación Sid@r. http://www.sidar.org/index.php

-Bobby Approval. http://www.cast.org/bobby

-Accessible Acrobat. http://access.adobe.com

\section{Normas y validación}

- $\mathrm{Html}$ validator. http://validator.w3.org/

—CSS validator. http://jigsaw.w3.org/css-validatorl

-Platform for internet content selection (Pics). http://www.w3.org/PICS/

- The DC Metadata Initiative-Government Application Profile. http://dublincore.org/documents/2001/09/I 7/ gov-application-profile/ 
Canals, Isidre. «Democràcia i internet: la contribució d'internet a la democràcia». En: Democracia.web, papers sobre democràcia, 2001. http://www.democraciaweb.org

\section{Ciberp@ís, 2001.}

http://www.ciberpais.elpais.es/d/20010705/cibersoc/portada.htm

COM 585. La información del sector público: un recurso clave para Europa. Libro Verde sobre la información del sector público en la sociedad de la información. Bruselas: Comisión Europea, 1998.

COM 607 final. La eEurope 2002: creación de un marco comunitario para la explotación de la información del sector público, 2001.

http://europa.eu.int/information_society/eeuropelaction_plan/pdf/actionpl an_en.pdf

COM 265 final. eEurope 2005: una sociedad de la información para todos, plan de acción que se presentó con vistas al Consejo Europeo de Sevilla, 21-22 de junio de 2002.

http://europa.eu.int/information society/eeurope/news library/documents/ eeurope2005/eeurope2005_es.p.pdf

Cornella, Alfons. «La información pública, contenidos esenciales en la sociedad de la información». En: Extranet, mensaje 403, 16-02-99.

Delgado García, Ana Mä; Oliver Cuello, Rafael. Nuevas tecnologías y declaraciones tributarias, portal UOC.

http://www.uoc.edu/web/esp/art/uoc/delgado0102/delgado0102_imp.html

E-accessibility: accessibility of public web sites and their content. Communication from the Commission to the Council, the European Parliament, the Economic and Social Committee, and the Committee of Regions, 2002.

http://europa.eu.int/information_society/eeurope/action_plan/eaccess/inde x_en.htm

eEurope: una sociedad de la información para todos. Comunicación sobre una iniciativa de la Comisión para el Consejo Europeo de Lisboa, 23 y 24 de marzo de 2000.

http://europa.eu.int/information_society/eeurope/news_library/pdffiles/in itiative_en.pdf

Eisenstadt, Marc; Vincent, Tom. The knowledge web: learning and collaborating on the net. Chapter 2. London: Knowledge Media Institute, 2000.

Electronic publishing services. Government information and the UK information market. Reino Unido: Department of trade and industry, mayo de 2000 .

Fundación Retevisión [Fundación Auna, desde mayo de 2002]. e-España 2002: situación de España ante la sociedad de la información, 2002. http://www.fundacionretevision.es/publi/publi_ee00.htm

Fundación Retevisión. Informe sobre la evolución de los servicios públicos electrónicos dentro de la Unión

Europea. Estudio encargado por la Presidencia Española del Consejo de la UE. Madrid, 25-26 abril 2002.

http://www.map.es/csi/pdfleGovEsp_definitivo.pdf

Guidance on the work of the government information service, 2001. http://www.gics.gov.uk/handbook/guidance/giswork/index.htm
Internet intelligence 2001. Politics online.

http://www.politicsonline.com/specialreports/010803/eusurvey2001.asp

Jones, Rhion. «What price CRM? government computing». En: CRM supplement, 2002, mayo, p. 6.

Liikanen, Erkki. EGovernment-providing better public service and wider participation for citizens. Stockholm, 29 June 2001, speech/01/319, [discurso].

http://europa.eu.int/rapid/

Marcos, Mari Carmen; Gil, Ana Belén. «Información parlamentaria autonómica en la Red: un impulso electrónico a la democracia». En: El profesional de la información, 2002, marzo-abril, v. 11, n. 2, pp. 91-101.

Murray, Sarah. «Online opportunity to transform administrations and services at all levels». En: Financial times, June 18, 2001. http://specials.ft.com/ftit/june2001/FT3IICMY2OC.html

Nielsen, Jakob. Alertbox on web usability. http://www.useit.com/alertbox

Nistal Rosique, Gloria. «El portal de las administraciones públicas para los ciudadanos y las empresas». En: El profesional de la información, 2002, marzo-abril, v. 11, n. 2, pp. 111-121.

«Radiografía de las 52 capitales españolas en internet». En: El país.es, Cibersociedad, 5 de julio de 2001.

http://www.ciberpais.elpais.es/20010705/cibersoc/portada.htm

Socitm insight. Better connected 2002?: a snapshot of all local authority websites, 2002.

http://www.socitm.gov.uk

Sydmonds, Matthew. «The next revolution: after e-commerce, get ready for e-government». En: Economist.com, 22 de junio, 2000.

http://www.economist.com

Usableweb-Ecommerce

http://usableweb.com/topics/000481-0-0.html

Vianello Osti, Marina; Sánchez Blanco, Emilio. «Estructura y accesibilidad de la información institucional en las páginas web de las comunidades autónomas españolas: análisis y caso práctico». En: IVII Jornadas españolas de documentación: la gestión del conocimiento, retos y soluciones de los profesionales de la información, 2000, pp. 369-381.

Web quality briefings 1: Case study of advertising on a government website (disponible en varios formatos).

http://www.e-envoy.gov.uk/oee/oee.nsf/sections/webqualitybrieftop/\$file/webqualityindex.htm

Pablo Lara Navarra, profesor coordinador del área de gestión de la información y conocimiento de la UOC. plara@uoc.edu

José Ángel Martínez Usero, consultor de la licenciatura en documentación de la UOC y profesor asociado de la Universidad Carlos III.

jmartinezu@uoc.edu

\section{Próximos números monográficos}

\section{Enero 2003 Perfil profesional y mercado de trabajo \\ Marzo 2003 Estándares documentales en internet: Z39.50, Dublin Core... \\ Mayo $2003 \quad$ Auditoría de la información}

Los interesados pueden remitir notas, artículos, propuestas, publicidad, comentarios, etc., sobre estos temas a: 